\title{
Investigation of droplet nucleation in CCS relevant systems - design and testing of the expansion chamber
}

\author{
Miroslav Čenský ${ }^{1, *}$, Jan Hrubý ${ }^{1}$, Václav Vinš ${ }^{1}$, Jiří Hykl $^{1}$ and Bohuslav Šmíd ${ }^{1}$ \\ ${ }^{1}$ Institute of Thermomechanics of the CAS, Dolejškova 1402/5, Prague 8, 182 00, Czech Republic
}

\begin{abstract}
A unique in-house designed experimental apparatus for investigation of nucleation of droplets in CCS relevant systems is being developed by the present team. The apparatus allows simulating various processes relevant to $\mathrm{CCS}$ technologies. Gaseous mixtures with $\mathrm{CO}_{2}$ are prepared in a Mixture Preparation Device (MPD) based on accurate adjustment of flow rates of individual components [EPJ Web of Conferences 143, 02140 (2017)]. The mixture then flows into an expansion chamber, where it undergoes a rapid adiabatic expansion. As a consequence of adiabatic cooling, the mixture becomes supersaturated and nucleation and simultaneous growth of droplets occurs. In this study, we describe the design and testing of the expansion part of the experimental setup. The rapid expansion was realized using two valve systems, one for low pressures (up to $0.7 \mathrm{MPa}$ ) and the other for high pressures (up to $10 \mathrm{MPa}$ ). A challenge for a proper design of the expansion system is avoiding acoustic oscillations. These can occur either in the mode of Helmholtz resonator, where the compressible gas in the chamber acts as a spring and the rapidly moving gas in the valve system as a mass, or in the "flute" mode, where acoustic waves are generated in a long outlet tubing.
\end{abstract}

\section{Introduction}

The team of the Laboratory of Phase Transition Kinetics at the Institute of Thermomechanics of the Czech Academy of Sciences (CAS) is developing a unique experimental apparatus for investing the formation of droplets in $\mathrm{CO}_{2}$-rich systems.

To obtain new valuable data for nucleation of droplets in binary and multicomponent mixtures important in the design of new Carbon Capture and Storage (CCS) technologies $[1,2]$ is the main motivation for this research. The apparatus allows for simulating droplet nucleation relevant for large variety of processes of technologies.

Gaseous mixtures with $\mathrm{CO}_{2}$ are prepared in a Mixture Preparation Device (MPD) based on an accurate adjustment of flow rates of individual components [1]. The mixture then flows into an expansion chamber, where it undergoes a rapid adiabatic expansion. As a consequence of adiabatic cooling, the mixture becomes supersaturated and nucleation and simultaneous growth of droplets occurs. The droplets are indicated optically based on laser light scattering and the nucleation and growth rates can be evaluated.

Expansion cloud chambers have been used for the research of droplet formation for more than a century [3] and many high-quality data sets for homogeneous nucleation rates have been obtained using the so-called nucleation pulse method implemented in expansion chambers $[4,5]$. In this method, nucleation occurs in a short period of time (typically $1 \mathrm{~ms}$ ) and the supersaturation is reduced afterwards such that the existing droplets can grow to observable sizes (diameter of several hundred nanometers), but practically no new droplets appear. This approach is, however, limited to systems, where the growth is slow enough. In case of nucleation in $\mathrm{CO}_{2}$ rich mixtures, the growth is very rapid. Therefore, rather than the nucleation pulse method [6], we apply the continuous expansion method [7]. Here, the main control parameter is the expansion rate. The expansion rate, together with the nucleation and growth kinetics, determines the maximum nucleation rate achieved in the process. The higher is the expansion rate, the higher is the maximum nucleation rate. In order to have a broad experimental window, it is necessary to have the possibility of adjusting the nucleation rate in a broad range.

New apparatus for investigation of nucleation in $\mathrm{CO}_{2}$-rich systems was described in our previous paper [1], which was focused on the $\mathrm{CO}_{2}$ branch of the mixture preparation device. In this study, we describe the design and testing of the expansion part of the experimental setup.

\section{Experimental Apparatus}

The rapid expansion was realized using two valve systems, one for low pressures (up to $0.7 \mathrm{MPa}$ ) and the other for high pressures (up to $10 \mathrm{MPa}$ ). A challenge for a proper design of the expansion system is avoiding acoustic oscillations. These can occur either in the mode of Helmholtz resonator, where the compressible gas in

\footnotetext{
Corresponding author: censky@it.cas.cz
} 
the chamber acts as a spring and the rapidly moving gas in the valve system as a mass, or in the "flute" mode, where acoustic waves are generated in a long outlet tubing. Small acoustic oscillations lower the accuracy of the experiments; large oscillations can completely distort the expansion and even lead to the malfunction of the piston (or diaphragm) valve, which repeatedly closes and opens.

The setup of low-pressure expansion system is shown in Figure 1 and of high-pressure expansion system in Figure 2. Both low and high-pressure apparatuses consist of high pressure expansion chamber (1) pressurized from gas vessel (11) by nitrogen which was used as a model gas in the tests performed in this study. The initial pressure in the expansion chamber was set with a precision back pressure regulator from Equilibar, LLC ${ }^{\text {a }}$ manufacturer, (12) equipped with manometer (13). Precise measurement of the initial pressure inside the expansion chamber and a rapid pressure drop during the expansion in both low and high pressure expansion systems are measured with fast-response pressure transducers HEM-375 from Kulite Semiconductor Products $^{\mathrm{b}}$ (3) available for pressure range up to 100,500 and 2500 PSI. Electric signal from pressure transducer is amplified and connected to terminal block BNC-2110 used as an interface to PC with multifunction I/O device PCI-6115 from National Instruments ${ }^{c}$ manufacturer.

The low-pressure expansion system (Fig. 1) consists of a pilot-operated diaphragm valve from ASCO Valve, Inc. ${ }^{d}$ (2) followed by a throttling valve (5). For very slow expansions, the low pressure system uses a metering needle valve from $\mathrm{HOKE}^{\mathrm{e}}$ (6) followed by a manually controlled solenoid valve ASCO $^{\mathrm{d}}$ (7).

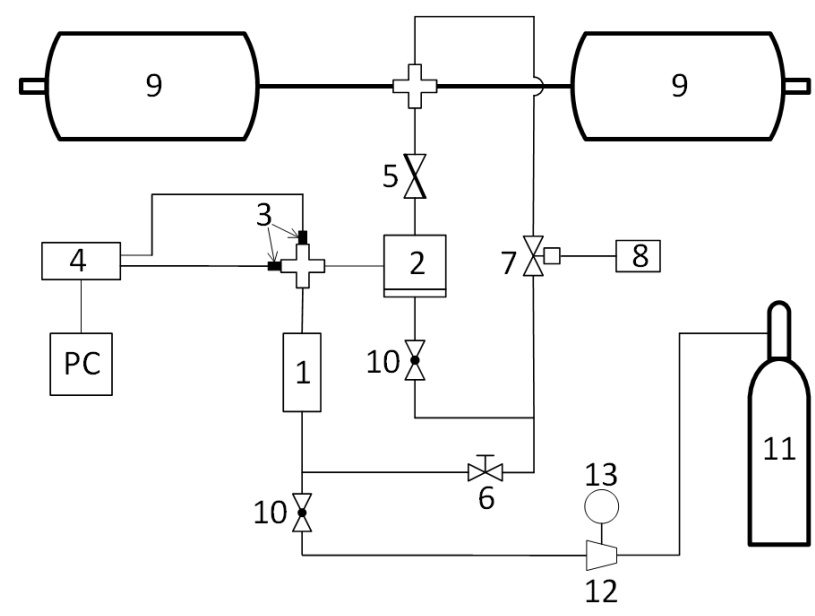

Fig. 1. Experimental apparatus for rapid expansion with throttle and needle valve. 1 - high pressure expansion chamber, 2 - pilot-operated diaphragm valve, 3 - rapid high pressure transducers, $4-$ BNC terminal block connected with PC, $5-$ throttling valve, 6 - metering needle valve, 7 - solenoid valve, 8 - solenoid valve controller, 9 - expansion vessels, 10 - ball

${ }^{a}$ www.equilibar.com

$\mathrm{b}$ www.kulite.com

${ }^{\circ}$ www.ni.com

$\mathrm{d}$ www.asco.com

${ }^{\mathrm{e}}$ www.hoke.com valves, 11 - gas vessel with nitrogen, 12 - pressure regulator, 13 - manometer.

The high-pressure system shown in Figure 2 is intended for investigation of most important $\mathrm{CO}_{2}$-rich mixtures relevant for the CCS technologies. The high-pressure setup was based on a commercially available pilotoperated piston valve from ASCO Valve, Inc. ${ }^{\mathrm{d}}$ manufacturer which was modified such that its saddle was directly followed by a throttling needle in order to minimize acoustic oscillations. The throttling needle has a special shape engineered such that the flow area can be reproducibly adjusted from fully open down to a fraction of percent. The adjustable position of the stem is measured with a digital distance indicator ID-H0530 from Mitutoyo ${ }^{f}$ manufacturer with micrometer resolution. Expanded gas is exhausted via two expansion vessels (9) opened to atmospheric pressure in the tests reported.

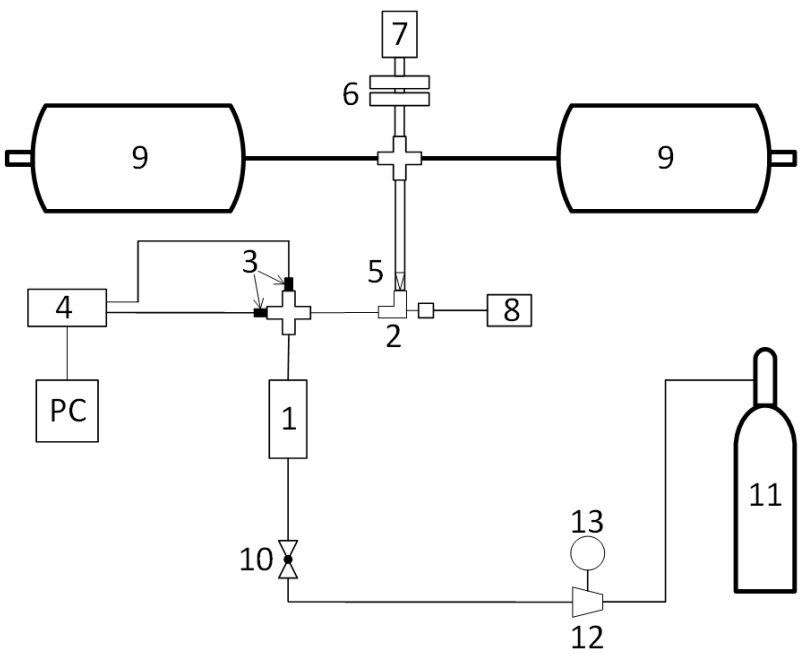

Fig. 2. Experimental apparatus for slow expansion special throttle valve. 1 - high pressure expansion chamber, 2 - pilotoperated piston valve, 3 - rapid high pressure transducers, $4-$ BNC terminal block connected with PC, 5 - throttling needle valve with a special shape, 6 - manual throttle valve controller, 7 - precise distance indicator, 8 - electromagnetic valve controller, 9 - expansion vessels, 10 - ball valves, 11 - gas vessel with nitrogen, 12 - pressure regulator, 13 - manometer.

\section{Results and Discussion}

Several series of expansions were done with nitrogen in both low and high pressure systems and corresponding expansion rates were evaluated based on following equation:

$$
\text { expansion rate }=\frac{d \ln p}{d t}=\frac{1}{p} \cdot \frac{d p}{d t}
$$

where $p$ is pressure in expansion chamber and $t$ is time. Figure 3 shows time dependence of pressure during the low-pressure slow expansion from approximately 700 $\mathrm{kPa}$ via the metering needle valve (6 in Figure 1). Higher

\footnotetext{
${ }^{\mathrm{f}}$ www.mitutoyo.com
} 
number of revolutions, given as rev. in the figure legend, is directly linked to wider opening of the needle valve. Expansion rate is evaluated from a slope of the pressure on time between pressures 400 and $500 \mathrm{kPa}$, where the pressure dependence is nearly linear. Dependence of the evaluated expansion rate on opening of the metering needle valve is shown in Figure 4.

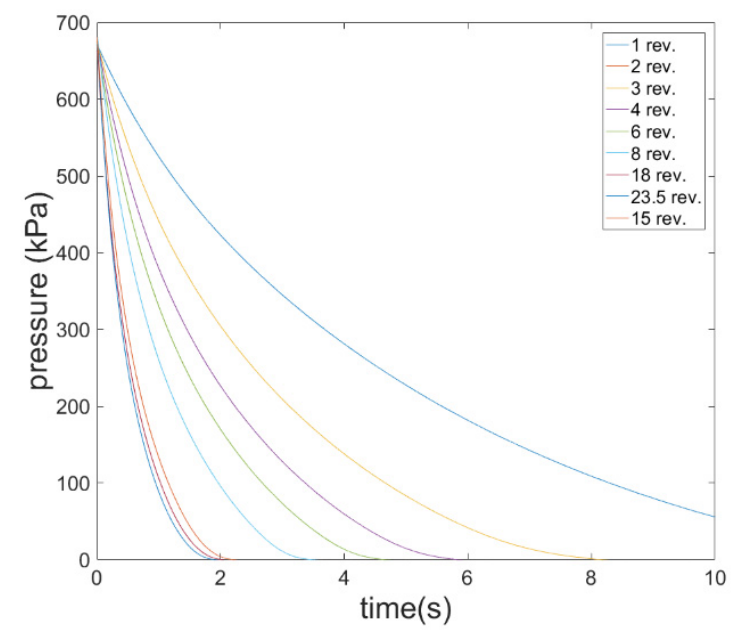

Fig. 3. Nitrogen pressure dependence on time during expansion from $700 \mathrm{kPa}$ through various openings of metering needle valve.

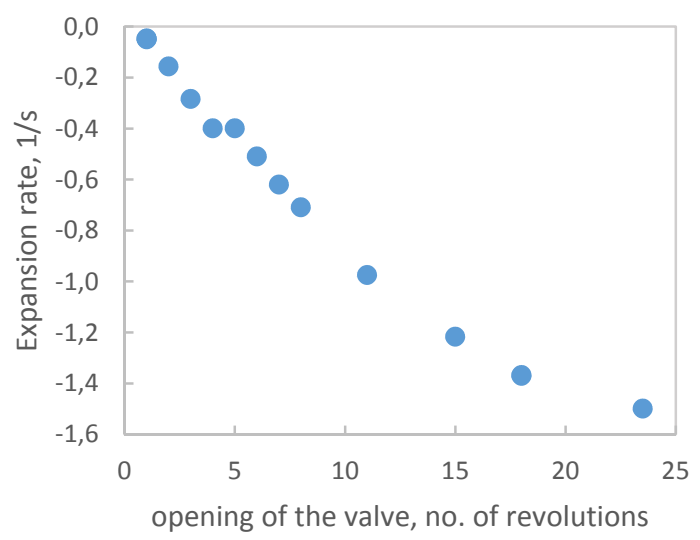

Fig. 4. Expansion rate dependence on opening of metering needle valve for expansion from $700 \mathrm{kPa}$. Slope of the expansion rate evaluated from linear interpolation between 400 and

$500 \mathrm{kPa}$.

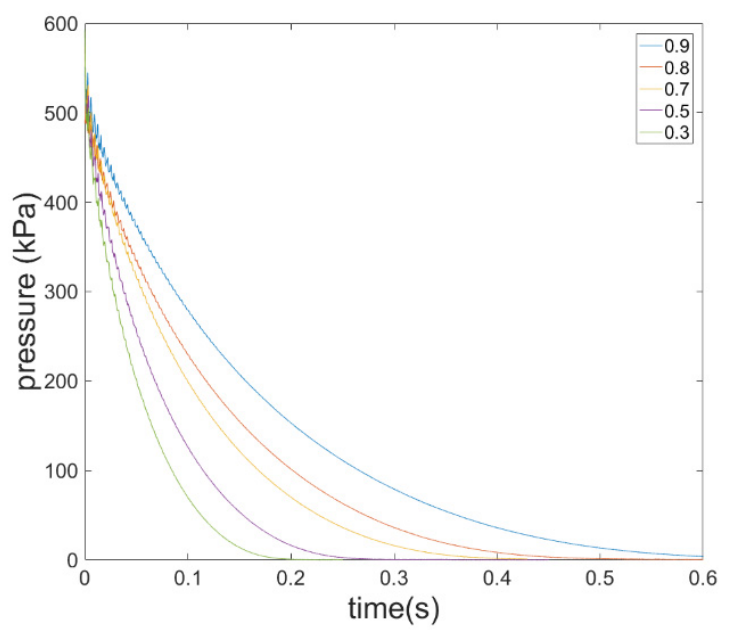

Fig. 5. Nitrogen pressure dependence on time during expansion from $600 \mathrm{kPa}$ through various small openings of throttling valve.

Time dependence of pressure during the low-pressure expansion from approximately $600 \mathrm{kPa}$ via the throttling valve ( 5 in Figure 1) is shown in Figures 5 and 6. Higher value of a relative number corresponds to wider opening of the valve and consequently to higher expansion rates. Expansion rates were evaluated from a slope of the pressure depending on time between pressures 400 and $500 \mathrm{kPa}$ where this dependence can be considered nearly linear. Dependence of the evaluated expansion rates on opening of the throttling valve is shown in Figure 7.

In the system with throttling valve, behaviour in the mode of Helmholtz resonator can be observed particularly in the beginning of the expansion, where generated acoustic waves caused oscillations of the pressure.

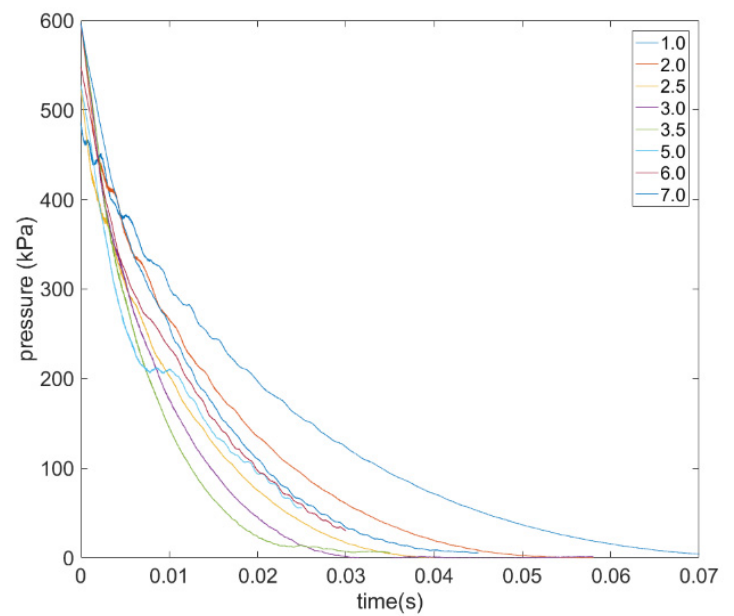

Fig. 6. Nitrogen pressure dependence on time during expansion from $600 \mathrm{kPa}$ through various wide openings of throttling valve. 


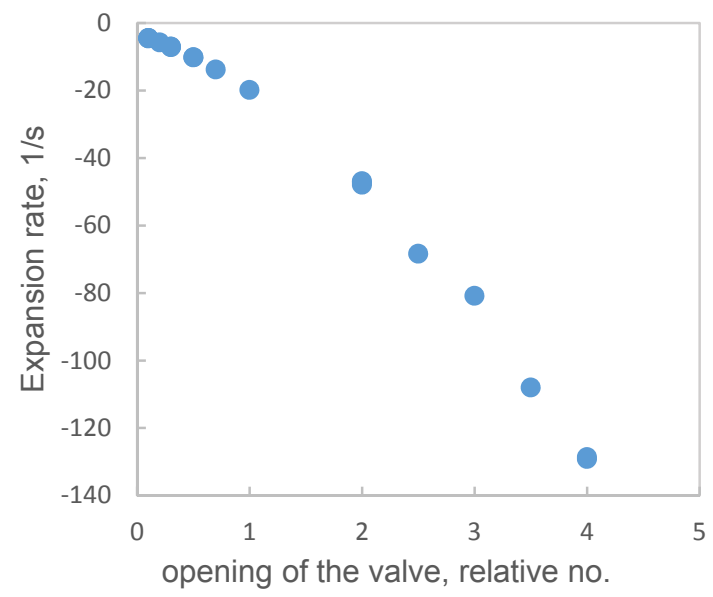

Fig. 7. Expansion rate dependence on opening of throttling valve for expansion from $600 \mathrm{kPa}$. Slope of the expansion rate evaluated from linear interpolation between 400 and $500 \mathrm{kPa}$.

The high-pressure system with the throttling needle valve with a special shape was tested for two initial pressures of 5 and $10 \mathrm{MPa}$.

Time dependences of pressure during the expansion from $5 \mathrm{MPa}$ and $10 \mathrm{MPa}$ via throttling needle valve with a special shape (5 in Figure 2) is shown in Figures 8 and 9 , respectively. Opening of the valve was measured precisely with digital distance indicator. Distance around $6.5 \mathrm{~mm}$ corresponds to the maximum opening of the valve. Expansion rates were evaluated from a nearly linear slope of the pressure on time in the pressure ranges of 4.5 to $4 \mathrm{MPa}$ and 9 to $8 \mathrm{MPa}$, respectively. Dependence of the evaluated expansion rates on opening of the throttling needle valve is shown in Figure 10.

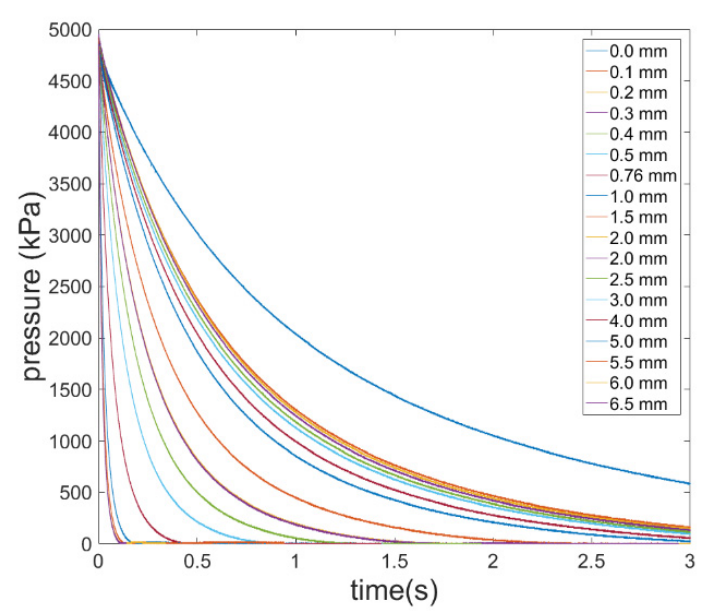

Fig. 8. Nitrogen pressure dependence on time during expansion from $5 \mathrm{MPa}$ through various openings of throttling needle valve with a special shape.

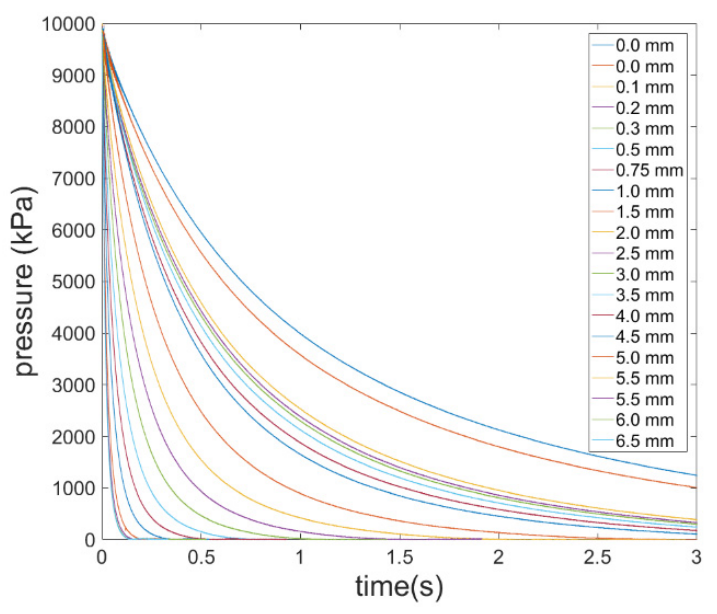

Fig. 9. Nitrogen pressure dependence on time during expansion from $10 \mathrm{MPa}$ through various openings of throttling needle valve with a special shape.

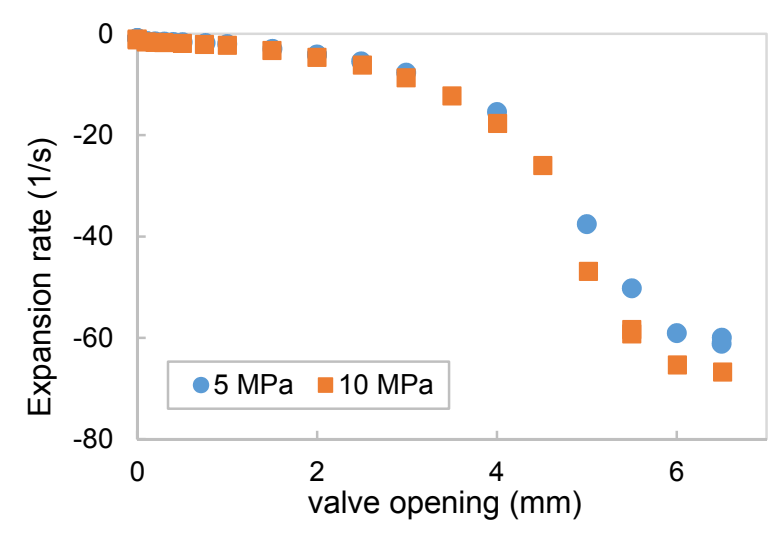

Fig. 10. Expansion rate dependence on opening of throttling needle valve with a special shape for expansion (item 5 in Figure 2) from 5 and $10 \mathrm{MPa}$. Slope of the expansion rate evaluated from linear interpolation between 9 and $8 \mathrm{MPa}$.

\section{Conclusions}

A droplet nucleation apparatus for CCS relevant systems is under construction in our laboratory. A newly designed expansion part of this apparatus was tested in this work. We used two arrangements for expansions in low and high pressure system using nitrogen as an expansion gas. The experiments showed that for the lowpressure system the expansion rate could be adjusted between 0.05 and $120 \mathrm{~s}^{-1}$ and for the high-pressure system the expansion rate could be adjusted between 0.87 and $67 \mathrm{~s}^{-1}$. It has been shown that these ranges of expansion rates can be achieved in the whole range of initial pressures considered, i.e. from 0.2 to $10 \mathrm{MPa}$.

\section{Acknowledgement}

The research leading to these results has received funding from the Norwegian Financial Mechanism 2009-2014 under Project Contract No. 7F14466. Additional experiments have been performed in frame of project No. 16-02647S by the Czech Science 
Foundation. Institutional support RVO:61388998 is also gratefully acknowledged.

\section{References}

1. V. Vinš, M. Čenský, J. Hykl, J. Hrubý: EPJ Web of Conferences 143, 02140 (2017)

2. IPCC Special Report: Carbon Dioxide Capture and Storage - Summary for Policymakers. Intergovernmental Panel on Climate Change, Eighth Session of IPCC Working Group III, Montreal, (Canada) 22-24 September 2005

3. C.R.T. Wilson: Phil. Trans. R. Soc. A., London 189, 265-307 (1897)

4. R.C. Miller, R.J. Anderson, J.L. Kassner, D.E. Hagen: J. Chem. Phys. 78, 3204-3211 (1983)

5. J. Wölk, R. Strey: J. Phys. Chem. B, 105, 11683$11701(2001)$

6. A. Fladerer, R. Strey: J. Chem. Phys. 124, 164710 (2006)

7. H.J.S. Smolders: Ph.D Thesis, Eindhoven University of Technology, (1992). 